\title{
Fingerprint Recognition Using Directional Micropattern Histograms and LVQ Networks
}

\author{
Sheng-De Wang and Chih-Jen Lee \\ Dept. of Electrical Engineering \\ National Taiwan University \\ Taipei, Taiwan \\ sdwang@hpc.ee.ntu.edu.tw
}

\begin{abstract}
The paper is about designing a fingerprint recognition system that makes use of the directional micropattern histograms of fingerprint image for local ridge orientation calculation, core point detection, and feature extraction. An improved learning vector quantization network is also proposed to avoid the unfairness of the winning rate and to determine a proper number of hidden units. Experimental results show that the recognition rate of the proposed method is $99.62 \%$ for a small-scale fingerprint database.
\end{abstract}

\section{Introduction}

Because of durability and uniqueness, fingerprints have been widely applied in several fields of personal identification including criminal identification and access control. However, a reliable and efficient fingerprint recognition system is difficult achieved, due to a number of factors such as different impression pressure, rotation, rolling, perturbation, epidermal condition, errors from acquisition devices, and problems of inked fingerprint (uneven, over or insufficient ink). Therefore, it is very important for fingerprint representation to endure above problems and still preserve sufficient information for recognition.

Fingerprints are full of ridge and valley structures. These structures preserve global information (ridge orientation) and local information (minutiae, including ridge endings and bifurcations). For a large-scale database, conventionally, fingerprint recognition is carried out at two different levels. At the coarse-level fingerprints are classified into some classes based on their global structure information $[3,4]$. The fine-level recognition is performed by comparing the minutiae sets between the input and the template patterns $[2,8]$.

The main steps for minutia-based fingerprint recognition are image acquisition, enhancement, ridge extraction, thinning, minutia detection, and matching [2, 8]. However, this approach is very sensitive to the noise and deformation. For instance, ridge endings may disappear because the finger is pressed too hard and bifurcations may also disappear for insufficient pressure [10]. Moreover, the acquisition equipment and the preprocessing steps may destroy or create false minutiae. In other words, the performance of minutia extraction algorithm relies heavily on the quality of input images and the accuracy of every preprocessing step. Nevertheless, fingerprint images are not always have well-defined ridge structures and the computations for thinning, minutia detection, and matching are expensive.

In this paper, we will propose using the directional micropattern histogram [4] to represent the ridge structures. The proposed fingerprint recognition method consists of the processing steps of image enhancement, binarization, feature extraction (including core point detection), and recognition, as shown in Fig. 1. It is no need for thinning, minutiae detection, and matching. At last, we will use a smallscale fingerprint database to test the performance of these features with LVQ networks.

\begin{tabular}{|c|c|c|c|}
\hline $\begin{array}{l}\text { Gray-level } \\
\text { image }\end{array}$ & $\begin{array}{l}\text { inarized } \\
\text { image }\end{array}$ & $\begin{array}{l}\text { Directional micr } \\
(7 \times 5,9 \times 7\end{array}$ & $\begin{array}{l}\text { tern histograms } \\
\times 9,13 \times 11)\end{array}$ \\
\hline $\begin{array}{c}\text { Enhancement } \\
\text { Binarization }\end{array}$ & $-\frac{C}{F}$ & $\begin{array}{l}\text { e point detection } \\
\text { ature extraction }\end{array}$ & $\begin{array}{c}\text { Recognition } \\
\text { (LVQ networks) }\end{array}$ \\
\hline
\end{tabular}

Figure 1. Block diagram of proposed method

\section{Preprocessing}

\subsection{Image acquisition}

For fingerprint acquisition, in general, there are two primary methods: inked (off-line) and inkless (on-line). The 
inkless method using a CCD-based device to capture fingerprint images is suitable for on-line fingerprint verification. Relatively, the inked fingerprint images can be more complicated because some problems may arise from shift, rotation, rolling and the ink pressure but are suitable for off-line fingerprint recognition. We are aimed to develop a recognition system for the inked fingerprint images.

In our database, we collected 288 inked fingerprint images from 12 persons ( 24 images per finger) and captured their digital format with a scanner at 200dpi and 256 graylevel resolution. Although NIST fingerprint databases [9] are sampled at 500dpi, fingerprint images can be recognized at $200 \mathrm{dpi}$ by human eyes. For a small-scale fingerprint recognition system, the use of low-resolution images is efficient and practicable.

\subsection{Fingerprint enhancement and binarization}

The ridge structures in the digitized fingerprint images are not always well defined. Therefore, many methods have been proposed to enhance the raw fingerprint images $[1,6]$. To obtain clear ridge structures efficiently, we apply the simple and fast method including smoothing, enhancement, smoothing and enhancement. After having the clear ridge structures, the binarized image is obtained by put the grayscale image through an adaptive threshold mean value. Although the result of this method is not the best for fingerprint enhancement, the binarized images can be obtained quickly and still preserve sufficient information for recognition. In [1], the use of Gabor filters to enhance the raw fingerprint image can obtain better ridge structures if the associated parameters are properly tuned. Otherwise, the enhancement function of Gabor filters may not be achieved, but the smoothing function may appear.

\section{Feature extraction}

\subsection{Directional micropattern histogram and local ridge orientation}

In a $2 \times 2$ binarized image, there are 16 micropatterns. Except the all white and all black, the micropatterns can be classified into five types, as shown in Fig. 2, corresponding to $0^{\circ}, 45^{\circ}, 90^{\circ}, 135^{\circ}$, and undefined directional components, respectively. Because the Type $\mathrm{V}$ may be $45^{\circ}, 135^{\circ}$ or noise, this type is discarded. The directional micropattern histogram, a histogram of four types of micropatterns, is obtained to represent the ridge structure of each $8 \times 8$ block. According to the directional micropattern histograms, the local ridge orientation of each block is computed as follows:

$$
\Theta=\frac{\sum_{k=1}^{4} h_{k} \times \theta_{k}}{\sum_{k=1}^{4} h_{k}}
$$

where $h_{k}$ is the histogram value of Type $k$ and $\theta_{k}=$ $\pi(k-1) / 4, k=1, \ldots, 4$. Except the representation of local ridge orientation, the directional micropattern histograms have more information than just the local ridge orientation. In Fig. 3, for example, their directional micropattern histograms are different: $(0,33,0,0),(0,48,0,0),(1,39,0,0)$, and $(2,31,5,1)$; but their local ridge orientations are similar: $45^{\circ}, 45^{\circ}, 43.9^{\circ}$, and $50.8^{\circ}$, respectively.

\begin{tabular}{|c|c|c|}
\hline 1 & $0^{0}$ & שם \\
\hline II & $45^{0}$ & 由曰ㅂ \\
\hline III & $90^{\circ}$ & 日 \\
\hline IV & $135^{\circ}$ & 由日田 \\
\hline v & $\begin{array}{l}\text { Don't } \\
\text { care }\end{array}$ & Q \\
\hline
\end{tabular}

Figure 2. Five types of micropatterns.

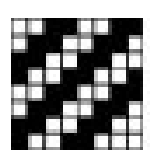

(a)

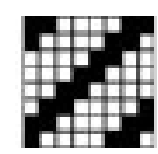

(b)

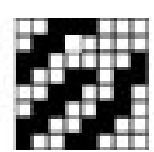

(c)

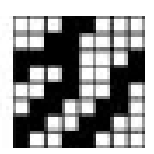

(d)
Figure 3. Some examples of the same orientation: (a) normal, (b) wide, (c) ridge ending, and (d) bifurcation.

is about

\subsection{Core point detection}

In the recognition stage, the comparison of two fingerprints must be based on the same reference point. The core point, the topmost point on the innermost upward recurving ridge, is the outstanding feature in fingerprints. We use this point as the reference point. Poincare index $[4,2]$ or structure information [7] can be applied to find the singular points including the core and delta points. However, these methods must compute the local ridge orientation and search the whole fingerprint image. To overcome the above problem, we develop a fast method to detect the core point by using directional micropattern histograms directly.

The following properties can be found by observing fingerprint images:

1. The intersections of the radii of curvatures for upper ridges are always near the core point. 
2. The directional micropattern histograms of the adjacent blocks of the core point block have the following properties: In the left block, the sum of the histogram values of $45^{\circ}$ and $90^{\circ}$ must be much larger than that of $135^{\circ}$. In the right block, similarly, the sum of the histogram values of $135^{\circ}$ and $90^{\circ}$ must be much larger than that of $45^{\circ}$. Moreover, the histogram value of $0^{\circ}$ of the upper-block must be much larger than that of the core point block. We can rewrite above properties as the following criteria:

$$
\begin{aligned}
h_{2}(x-1, y)+h_{3}(x-1, y) & \gg h_{4}(x-1, y)(2) \\
h_{4}(x+1, y)+h_{3}(x+1, y) & \gg h_{2}(x+1, y)(3) \\
h_{1}(x, y-1) & \gg h_{1}(x, y)
\end{aligned}
$$

According to above properties, we develop a fast algorithm to detect the core point:

1. Sample some points in the upper part of the fingerprint and find the average of the points at the intersections of the radii of curvatures.

2. Search the neighborhood of the intersection and use Eq. 2-4 to find the core point block.

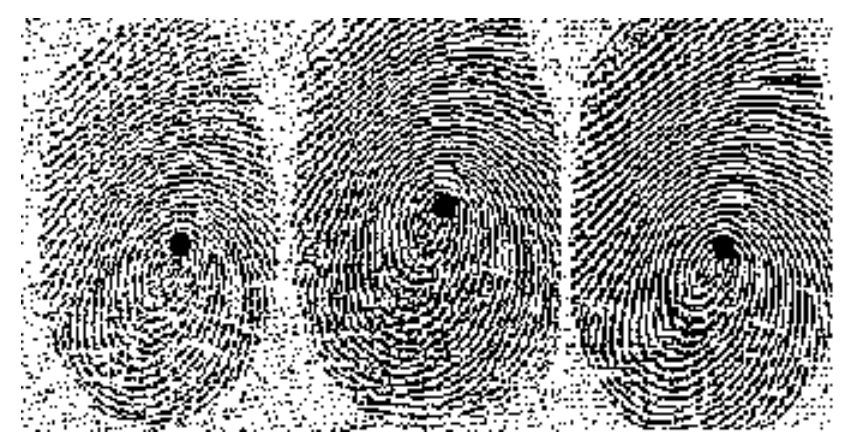

(a)
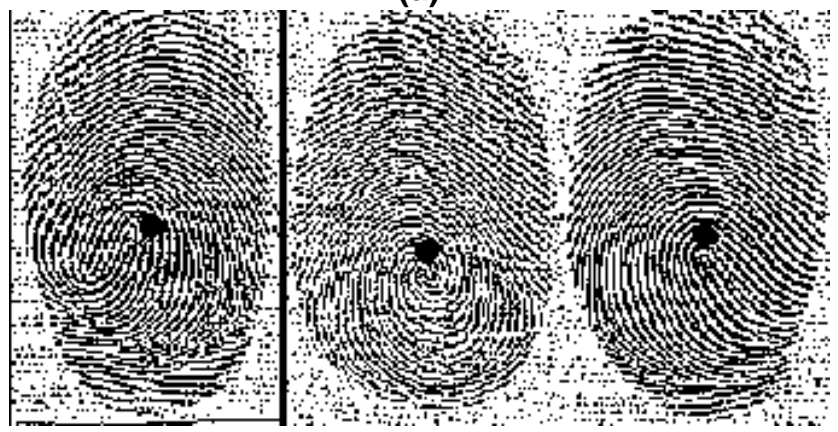

(b)

Figure 4. Detected core points: (a) the same and (b) different fingerprint patterns.

Fig. 4 shows some core points detected by the proposed method. The detected core points are central to the forma- tion of feature vectors. The directional micropattern histograms of some fixed image blocks around the core points will be used as the input feature vectors for the neural network decision stage. According to the core point, we divide the fingerprint image into a set of $8 \times 8$ non-overlapping blocks. To compare the influences of different sizes of input feature vectors, we use several different number of image blocks, namely, $7 \times 5,9 \times 7,11 \times 9$, and $13 \times 11$ blocks, as chosen from around the core point. Therefore, the size of the input feature vectors are $7 \times 20,9 \times 28,11 \times 36$, and $13 \times 44$, respectively.

\section{Fingerprint recognition using LVQ net- works}

We select learning vector quantization (LVQ) [5] as the recognition mechanism. LVQ networks can approximate the training set by a representative set of codebook vectors. During the training process, the problem of the unfairness of the wining rate in the hidden layer may occur. We propose the following modified learning vector quantization to improve the training process. Suppose that codebook vector $w_{C}$ is the closest to the input vector $x_{i}$ and $w_{D}$ is the corresponding one to the desired output. The proposed learning algorithm for the codebook vectors $w$ is described as follows:

If $x_{i}$ and $w_{C}$ belong to the same class,

$$
w_{C}(n+1)=w_{C}(n)-\eta_{1}(n)\left(x_{i}-w_{C}(n)\right) .
$$

If $x_{i}$ and $w_{C}$ belong to different classes,

$$
\begin{aligned}
& w_{C}(n+1)=w_{C}(n)-\eta_{1}(n)\left(x_{i}-w_{C}(n)\right), \\
& w_{D}(n+1)=w_{D}(n)+\eta_{2}(n)\left(x_{i}-w_{D}(n)\right),
\end{aligned}
$$

where $0<\eta_{1}(n), \eta_{2}(n)<1$ and decreases monotonically with the number of iterations $n$. The number of the hidden layer units in LVQ, in general, is obtained empirically. The following simple algorithm can be used to help determine the number of the hidden layer units.

1. Assign each unit of hidden layer corresponding to each class and train the network until it converges.

2. Stop learning if this network can recognize all training patterns. Otherwise, add one hidden layer unit corresponding to the desired output class of the training pattern which cannot be recognized and its codebook vector is set the same as the training pattern. Retrain the network and repeat this step.

\section{Experimental results}

Two training sets are used: the first one is with one pattern per class and the second one with two patterns per class. 
The remaining patterns are put to the test set. In the LVQ network, as shown in Fig. 5, the initial number of the hidden layer units are set equal to that of the classes. The recognition results with no reject option are shown in Table 1.

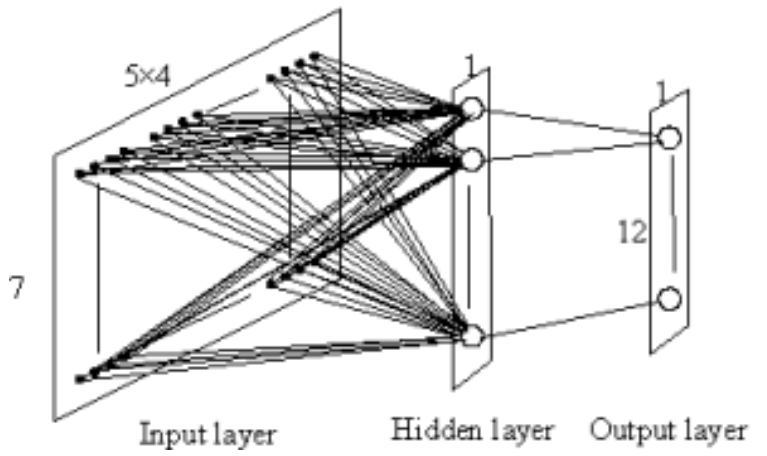

Figure 5. LVQ networks for $7 \times 5 \times 4$ input vectors.

Table 1. Recognition rate.

\begin{tabular}{|c|c|c|c|c|}
\hline Training set & $7 \times 5$ & $9 \times 7$ & $11 \times 9$ & $13 \times 11$ \\
\hline \hline 1 & 80.68 & 89.02 & 92.70 & 96.21 \\
\hline 2 & 87.88 & 93.56 & 98.11 & 99.62 \\
\hline
\end{tabular}

From Table 1, we find that the accuracy can reach more than $90 \%$ when the block number is more than $9 \times 7$, and even $99.62 \%$ for $13 \times 11$ in the second training set. The proposed approach using the directional micropattern histogram features is very suitable for ridge structures representation in fingerprints. It also has the advantage of using only one or two training patterns per class.

The purpose of the next experiment is to know how many units of the hidden layer per class are needed when the accuracy reaches $100 \%$ for our fingerprint database. The experimental results are shown in Table 2. We find that the LVQ network can adaptively grow the hidden layer units and need only one or two hidden layer units per class when the block number is more than $9 \times 7$ for 100 \% accuracy. If two classes are alike, more than one hidden unints are needed for discriminating each other in detail, such as class B and $K$. From above results, we found that the feature space of directional micropattern histograms around the core point is very promising for fingerprint ridge structures representation.

\section{Conclusion and future work}

We have successfully applied directional micropattern histogram features and LVQ to fingerprint recognition. The
Table 2. No. of hidden layer units per class.

\begin{tabular}{|c|c|c|c|c|c|c|c|c|c|c|c|c|}
\hline Feature size & $\mathrm{A}$ & B & & $D$ & & & & $\mathrm{H}$ & & & & Total \\
\hline$\overline{7 \times 5}$ & 3 & 3 & 3 & $1 \mid$ & 5 & & 2 & 2 & & $\overline{2}$ & 1 & $\overline{277}$ \\
\hline $9 \times 7$ & 2 & 2 & 2 & 1 & 1 & 2 & 2 & 1 & 1 & 2 & 1 & 19 \\
\hline $11 \times 9$ & 1 & 2 & 2 & 1 & 1 & 1 & 1 & 1 & 1 & 2 & 1 & 15 \\
\hline $13 \times 11$ & 1 & 2 & & 1 & 1 & 1 & 1 & 1 & 1 & $\overline{2}$ & $\overline{1}$ & 14 \\
\hline
\end{tabular}

performance of the proposed method can reach $99.62 \%$ accuracy when testing a small-scale fingerprint database that was sampled at only 200dpi with two training patterns per class. The representation of ridge structures using directional micropattern histograms is promising in facilitating efficient preprocessing and is robust in low image quality. The directional micropattern histogram features can also be applied to local ridge orientation computation and to the core point detection. Our future works are to develop an algorithm to directly extract directional information from gray-scale fingerprint image and test the algorithm with a large-scale fingerprint database.

\section{References}

[1] L. Hong, Y. Wan, and A. K. Jain. Fingerprint image enhancement: algorithms and performance evaluation. IEEE Trans. Pattern Analysis Machine Intelligent, 20(8):777-789, 1998.

[2] A. K. Jain, L. Hong, and R. Bolle. On-line fingerprint verification. IEEE Trans. Pattern Analysis Machine Intelligent, 19(4):302-314, 1997.

[3] K. Karu and A. K. Jain. Fingerprint classification. Pattern Recognition, 29(3):389-404, 1996.

[4] M. Kawagoe and A. Tojo. Fingerprint pattern classification. Pattern Recognition, 17(3):295-303, 1984.

[5] T. Kohonen. An instruction to neural computing. Neural Networks, 1:3-16, 1988.

[6] B. G. Sherlock, D. M. Monro, and K. Millard. Fingerprint enhancement by directional fourier filtering. IEE Proceedings-Vision, Image and Signal Processing, 141(2):87-94, 1994.

[7] V. S. Srinivasan and N. N. Murthy. Detection of singular points in fingerprint images. Pattern Recognition, 25(2):139-153, 1992.

[8] A. Wahab and S. H. Chin. Novel approach to automated fingerprint recognition. IEE Proceedings-Vision, Image and Signal Processing, 145(3):160-166, 1998.

[9] C. I. Watson. NIST Special Database 9: Mated Fingerprint Card Pairs. National Institute of Standards and Technology, May 1993.

[10] Q. Xiao and H. Raafat. Fingerprint image postprocessing: a combined statistical and structural approach. Pattern Recognition, 24(10):985-992, 1991. 